\title{
Value of Phenylalanine in Amniotic Fluid Detected by MS/MS for Screening of ma ternal PKU in the Second-Trimester
}

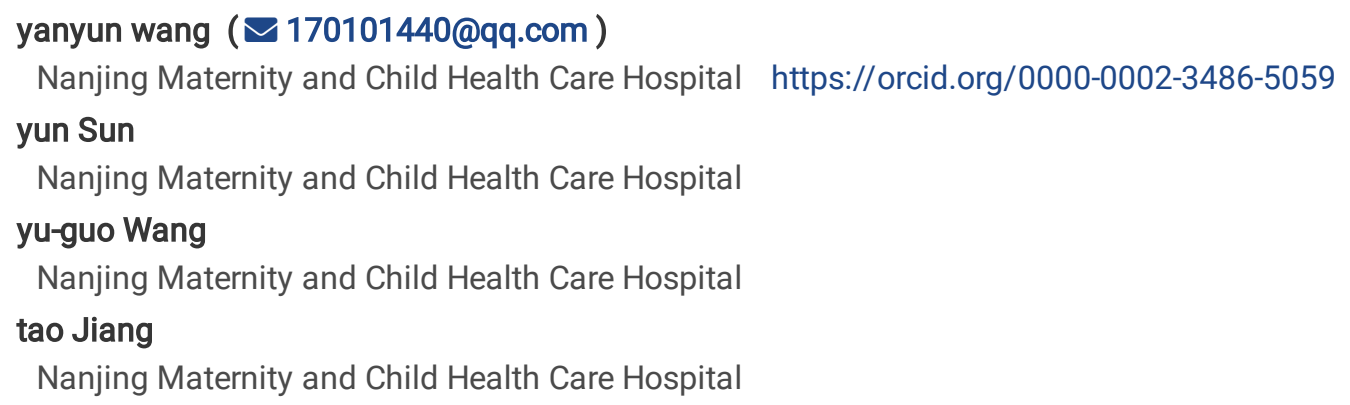

\section{Research}

Keywords: Maternal phenylketonuria (MPKU), phenylalanine (Phe), amniotic fluid (AF), tandem mass spectrometry (MS/MS), secondtrimester

Posted Date: July 22nd, 2020

DOI: https://doi.org/10.21203/rs.3.rs-42259/v1

License: (c) (i) This work is licensed under a Creative Commons Attribution 4.0 International License. Read Full License 


\section{Abstract}

Background: Phenylketonuria is the most common inherited metabolism disease in China. This study aimed to identify a new, sensitive, simple, and easy method of screening maternal Phenylketonuria in the fetal stage.

Methods: Samples of amniotic fluid, dried blood spots, and blood collected on the same day were obtained from women in the secondtrimester (16-28 weeks). Women had to meet the following criteria: (i) isolated high risk of quadruple marker screening or cell-free DNA screening, (ii) women who gave birth to at least one child with intellectual disability of unknown etiology, and (iii) women who gave birth to a child who died young from unknown etiology. The exclusion criteria were as follows: clinically evident chorioamnionitis, multiple gestation, and laboratory signs of infection in the amniotic fluid sample. Phenylalanine levels were measured using tandem mass spectrometry. Women who met criterion (i) served as controls whose results were used to set reference values of Phenylalanine in amniotic fluid. Women who met criteria (ii) and (iii) served as the case group whose results were used to check for maternal Phenylketonuria. The Spearman rank correlation test was used to analyze the correlation of Phenylalanine in amniotic fluid and in venous blood.

Results: We analyzed 365 samples of amniotic fluid. Among them, 345 were included in the control group, with reference values of Phenylalanine in amniotic fluid of 10.79-48.47 $\mu \mathrm{mol} / \mathrm{L}$. Twenty cases were included in the case group. One woman in the case group was diagnosed with hyperphenylalaninemia whose fetus was diagnosed with maternal Phenylketonuria by comprehensive analysis of Phenylalanine in dried blood spots and in amniotic fluid, head circumference in fetal ultrasound, and a previous history of adverse pregnancy. The correlation between Phenylalanine concentrations in venous blood and in amniotic fluid was weak.

Conclusions: Phenylalanine can be readily measured in amniotic fluid. Relative to controls, amniotic fluid levels of Phenylalanine was increased in maternal Phenylketonuria. These results can support the use of Phenylalanine as a screening tool for maternal Phenylketonuria. The significance of these change requires further study.

\section{Plain English Summary}

PKU screening began in 1985 in China『and was extended nationally in 1990. During this period, many baby with PKU did not received newborn screening. Now Women suffered with PKU who were born around 1990 and had not been diagnosed have entered the reproductive age. Most Obstetricians still lacked of the concept in inherited metabolism diseases囚including PKU and maternal PKU区, so it is not easy for such pregnant women to obtain diagnosis before delivery.

Non-specific amniotic fluid (AF) exfoliative cytological analysis are usually fetal chromosomes and CNV. Due to the variety of inherited metabolism diseases (IMDs) \this can lead to a large number of missed diagnosis of IMD fetus囚including maternal PKU, MMA and PAX.

In our research, we found that high PHE was detected in one AF sample by MS/MS. Then the clinical data of this woman were retrospectively analyzed. The woman was determined as mentally retarded after birth, did not receive regular medical treatment, and was unable to be enrolled in school. On the day of amniocentesis $\left(26^{+4}\right.$ weeks), an abdominal ultrasound showed that the biparietal diameter was $5.98 \mathrm{~cm}$ (reference interval: $6.98 \pm 0.57 \mathrm{~cm}$ ), and the head circumference was $22.19 \mathrm{~cm}$ (reference interval: $27.3 \pm 1.8 \mathrm{~cm}$ ). These findings suggested that the fetus had microcephaly with a head circumference of less than the third percentile. The pregnant woman gave birth to a girl in 2015. Her daughter is 2 years old, is unstable in walking, mentally retarded, and retarded in language and can only say "grandma". It is not surprising that Chromosome and chromosomal microarray analysis (CMA) test results of this fetus were normal. We have reason to believe that both the girl and the fetus are maternal PKU patients.

AF metabolite detection can not only detect maternal PKU囚but also diagnose MMA, PA and other organic acidemia in uterus. In my opinion, AF metabolite detection is a useful supplement to chromosomes and CNV whic has the advantage of no routine testing influence, speedy and low costs.

\section{Introduction}

Phenylketonuria (PKU) is the most common inherited metabolism disease (IMD) in China. The screening of neonatal PKU disease began in the 1960s in European and U.S.A but only started in the 1980s in China. During the process of gradually promoting PKU screening in China, some patients with PKU were not screened, diagnosed, and treated in time. These patients have gradually reached 
childbearing age. Therefore, avoiding newborn disabilities due to maternal phenylketonuria (MPKU) is particularly important. Rouse ${ }^{[1]}$ showed that the level of phenylalanine (Phe) in amniotic fluid (AF) is equivalent to that in blood, leading to fetal malformation and irreversible damage to the fetus. At present, the main purpose of research is to enable people who choose to prenatal screening so to avoid the birth of a child with IMDs, such as PKU or MPKU.

Maternal metabolic congenital malformation refers to damage to the embryo or fetus by abnormal metabolic substances before conception or during pregnancy. Classical diseases include maternal diabetes ${ }^{[2]}$, MPKU, and fetal alcohol syndrome ${ }^{[3]}$. MPKU is due to abnormal accumulation of Phe in the blood of pregnant women who have PKU before pregnancy. Additionally, in MPKU, PKU is not treated or the condition is not effectively controlled after treatment, leading to serious consequences such as brain damage, heart malformation, and head malformation of the intrauterine fetus ${ }^{[4-7]}$. Tandem mass spectrometry (MS/MS) is used to rapidly and accurately detect Phe content in AF. This study aimed to examine the feasibility of metabolic screening in AF, which might be considered as an additional biochemical test in pregnant women, especially for MPKU.

\section{Materials And Methods}

\section{Subjects}

From March 2018 to June 2018, a total of 365 pregnant women were enrolled in this study. The women were voluntarily included in a study at Nanjing Maternity and Child Health Care Hospital, Center for Genetic Medicine. The study was approved by the Nanjing Maternity and Child Health Care Hospital Ethics Committee. Written consent was obtained from the women before amniocentesis.

Samples of AF, dried blood spots (DBSs), and blood collected on the same day were obtained from women during the middle of pregnancy (16-28 weeks' gestation). Women who met the following criteria were included: (i) isolated high risk of quadruple marker screening or cell-free DNA screening ( $n=345)$, (ii)women who gave birth to at least one child with intellectual disability of unknown etiology ( $n=17)$, and (iii) women who gave birth to a child who died young from unknown etiology $(n=3)$. The exclusion criteria were as follows: clinically evident chorioamnionitis, multiple gestation, and laboratory signs of infection in the AF sample. Women who met criterion (i) served as controls whose results were used to set reference values of Phe in AF. Women who met criteria (ii) and (iii) served as the case group whose results were used to check for MPKU. Phe was detected in DBSs and AF using MS/MS.

\section{Sample collection}

A total of $4 \mathrm{ml}$ of venous blood was collected from pregnant women on the day of amniocentesis. The blood was placed into EDTA-2K tubes, and $50 \mu$ l of venous blood was dripped onto blank filter paper and dried in the dark to create DBS filters.

Dried AF spots were prepared as follows. The pregnant women were subjected to amniocentesis guided by B-ultrasound at 16-28 weeks of gestation. A volume of $20 \mathrm{ml}$ of AF was extracted; $5 \mathrm{ml}$ of AF was used for culture and $15 \mathrm{ml}$ of AF was used for chromosomal microarray detection. The AF sample used for chromosomal microarray was centrifuged at $1800 \mathrm{rpm}$ for $10 \mathrm{~min}$. A total of $50 \mu$ supernatant was dripped onto a blank filter paper, dried in the dark, and used for MS/MS detection. For detection of Phe in DBSs and dry AF spots, $3.2 \mathrm{~mm}$ of dry blood/AF filter paper was placed into a 96-well U-shaped plate. Using non-derivatization pretreatment (NeoBaseTM non-derivatization MS/MS kit; Perkin-Elmer, U.S.A), $100 \mu$ l of extract fluid and internal standard mixture was added to each well and shaken while being sealed at $45^{\circ} \mathrm{C}$ for $45 \mathrm{~min}$. The extracted fluid was then transferred to a 96 -well V-plate, which was covered with aluminum film and allowed to sit for $2 \mathrm{~h}$ at room temperature before detection.

\section{Statistical analysis}

Reference intervals of Phe in AF were established on the basis of analysis of Phe detection results of AF samples in the control group by MS/MS. SPSS 17.0 software was used for regression analysis of Phe concentrations in venous blood and in AF of 32 cases. The Spearman rank correlation was used as a measure of an association between Phe in venous blood and $A F$, and $P<0.05$ was considered statistically significant.

\section{Results}

Reference intervals of Phe in AF as detected by MS/MS 
A frequency distribution histogram of Phe showed that Phe levels showed a normal distribution (Figure 1). To determine cutoff values for Phe, we used the percentile method, so the $0.5^{\text {th }}$ and $99.5^{\text {th }}$ percentiles were calculated. The reference intervals of Phe was 10.79$48.47 \mu \mathrm{mol} / \mathrm{L}$ (Table 1).

\section{Phe values of $\mathrm{AF}$ in the case group}

Phe values in AF in 19 cases showed no obvious abnormalities. In the remaining case, the Phe value in AF was $390.27 \mu \mathrm{mol} / \mathrm{L}$, which was significantly higher than the reference interval. The Phe level in DBSs was $1108.14 \mu \mathrm{mol} / \mathrm{L}$ (reference interval: $25-90 \mu \mathrm{mol} / \mathrm{L}$ ) in this case. The clinical data of this woman with hyperphenylalaninemia (HPA) were retrospectively analyzed. The woman was determined as mentally retarded after birth, did not receive regular medical treatment, and was unable to be enrolled in school. On the day of amniocentesis $\left(26^{+4}\right.$ weeks), an abdominal ultrasound showed that the biparietal diameter was $5.98 \mathrm{~cm}$ (reference interval: $6.98 \pm 0.57 \mathrm{~cm}$ ), and the head circumference was $22.19 \mathrm{~cm}$ (reference interval: $27.3 \pm 1.8 \mathrm{~cm}$ ). These findings suggested that the fetus had microcephaly with a head circumference of less than the third percentile.

The pregnant woman gave birth to a girl in 2015. Her daughter is 2 years old, is unstable in walking, mentally retarded, and retarded in language and can only say "grandma". Because the pregnant woman had HPA, the girl was highly suspected as having MPKU.

However, the pregnant woman refused to accept further clinical interventions, including genetic testing. Chromosome and chromosomal microarray analysis (CMA) test results of the fetus were normal.

\section{Regression analysis of Phe concentrations in AF and in venous blood}

A frequency distribution histogram of Phe showed skewed distribution in AF. Therefore, the Spearman rank correlation test was used to analyze the correlation of Phe concentrations in AF and in venous blood. The correlation coefficient of Phe was $0.062(\mathrm{P}>0.05)(\mathrm{Figure}$ 2). This result suggested that the correlation between Phe concentrations in venous blood and AF was weak.

\section{Discussion}

MPKU syndrome was first described more than 60 years previously ${ }^{[8]}$. There is a convincing correlation between high maternal blood Phe concentrations, particularly during critical periods of embryogenesis in early pregnancy, and fetal abnormalities, including fetal growth retardation, microcephaly, and structural heart defects.

Phe in the blood of PKU pregnant woman enters the placenta through active transport. Persistently high Phe concentrations in the blood are toxic to development of fetal organs, especially for the brain and heart. A previous study showed that $92 \%$ of infants born by pregnant women with poorly controlled Phe levels in the blood had retarded development, $73 \%$ had microcephaly, and $12 \%$ had congenital heart disease ${ }^{[9]}$. However, if Phe levels in the blood are strictly controlled between 120 and $360 \mu \mathrm{mol} / \mathrm{L}$ from pre-pregnancy throughout pregnancy, the incidence of microcephaly can be reduced to $3 \%-8 \%{ }^{[9-10]}$.

The rates of Chinese national screening coverage in 2006 and 2013 were $39.1 \%{ }^{[11]}$ and $86.3 \%[12]$, respectively. According to the latest statistics from the Maternal and Child Health Information System (MCHIS) in 2016, the national neonatal screening coverage for Phe is just $96.6 \%$. With development of screening, diagnosis, and treatment policies, the Phe screening rate should become close to $100 \%$, and the incidence of MPKU could be reduced. However, before the full coverage of neonatal screening, some patients with PKU were not diagnosed and treated in a timely manner because of regional and economic social factors. When these patients reach childbearing age, MPKU is likely to occur, with frequent occurrence of a poor pregnancy history. Therefore, we hope to develop a new method to diagnose these patients.

Currently, common methods for investigating metabolites in AF include gas chromatography, liquid chromatography/MS, and ultrahigh performance liquid chromatography/MS. MS/MS has been used for prenatal diagnosis of methylmalonic acidemia by detecting $\mathrm{C} 3$ in AF. Additionally, MS/MS is easier to operate than gas chromatography. AF spot filter preparation is simple and only involves supernatant waste liquid after centrifugation, and detection does not interfere with routine detection of fetal chromosomes. Our center uses MS/MS to detect Phe concentrations in AF.

In this study, Phe concentrations in AF of pregnant women without PKU ranged from 10.79-48.47 $\mu \mathrm{mol} / \mathrm{L}$. One woman was diagnosed with HPA whose Phe level in AF was as high as $390.27 \mu \mathrm{mol} / \mathrm{L}$. Taking into account fetal biparietal diameter and head circumference, her fetus was diagnosed with MPKU. 
On the basis of a previous study ${ }^{[1]}$, which showed that the concentration of Phe in AF is equivalent to that in the venous blood of pregnant women, we further analyzed the relationship between Phe concentrations in venous blood and in AF. We found that the correlation of Phe in venous blood and in AF was not strong and is inconsistent with the conclusion of Rouse's study ${ }^{[1]}$. We believe that this discrepancy between studies may be due to the difference in detection methods used because Rouse used the gas chromatography method ${ }^{[1]}$ while we used the MS/MS method.

Although only one positive case was found in this study, an increasing amount of patients may be found with an increase in the number of amniocentesis. Additionally, our study suggests that prenatal diagnosis in amniocentesis should not be limited to conventional methods, such as chromosome analysis and CMA. Detection of metabolites in AF does not increase the pain of pregnant women, and the cost of detection is low. This detection of metabolites can be used to screen for methylmalonic acidemia, MPKU, and other IMDs, which is worth the attention of genetic doctors.

\section{Conclusions}

With improvement of medical awareness, some pregnant women with a poor pregnancy history request amniocentesis to improve fetus-related tests. If a pregnant woman has a genetic metabolic disease, such as PKU, and is pregnant without regular treatment, metabolites will affect the fetus, resulting in problems such as small head deformity and stillbirth. Additionally, some families of patients with PKU do not understand MPKU and may conceal the medical history. Therefore, for pregnant women who request amniocentesis, Phe concentrations in AF can be detected simultaneously to facilitate the diagnosis of MPKU. We hope to extend the mehthods of detecting other amino acid or acylcarnitine to the diagnosis of other IEMs from dried AF samples in the near future.

\section{Abbreviations}

MPKU

Maternal phenylketonuria; Phe:phenylalanine;AF:amniotic fluid; MS/MS:tandem mass spectrometry; IEMs:inherited metabolism diseases

\section{Declarations}

\section{Consent for publication}

Not applicable

\section{Availability of data and materials}

Data and Material available on request

\section{Conflicts of interest statement}

The authors declare that the research was conducted in the absence of any commercial or financial relationships that could be construed as potential conflicts of interest.

\section{Author contribution statement}

WYY, SY, and WYG conceptualized and designed the study; WYY performed the review process, drafted the initial manuscript, and extracted the data; SY reviewed the literature; WYG collected the amniotic fluid samples; and JT analyzed and interpreted the data. All authors made substantial contributions to revising the manuscript. JT is responsible for the overall content. All authors read and approved the final manuscript.

\section{Acknowledgments}

We sincerely thank all of the family members for their participation and cooperation in this study. We thank Ellen Knapp, PhD, from Liwen Bianji, Edanz Group China (www.liwenbianji.cn/ac), for editing the English text of a draft of this manuscript.

\section{Ethics statement}


This study was approved by the local Ethics Committee of Nanjing Maternity and Child Health Care Hospital. Informed written consent was obtained from all patients before enrollment in this study.

\section{Conflict of interest statement}

The authors declare that there are no conflicts of interest.

\section{Funding}

This was independent work and received no funding.

\section{References}

1. Rouse B, Azen C, Koch R, Matalon R, Hanley W, de la Cruz F, et al. Maternal phenylketonuria collaborative study (MPKUCS) offspring: Facial anomalies, malformations, and early neurological sequelae[J]. Am J Med Genet. 1997;69(1):89-95.

2. Jensen DM, Damm P, Moelsted-Pedersen L, Ovesen P, Westergaard JG, Moeller M, et al. Outcomes in type 1 diabetic pregnancies: a nationwide, population-based study[J]. Diabetes Care. 2004;27(12):2819-23.

3. Wattendorf DJ, Muenke M. Fetal alcohol spectrum disorders.[J]. Encyclopedia of Infant Early Childhood Development. 2008;16(6):533-44.

4. Koch R, Hanley W, Levy H, Matalon K, Matalon R, Rouse B, et al. The Maternal Phenylketonuria International Study: 1984-2002[J]. Pediatrics. 2003;112(6 Pt 2):1523-9.

5. Lenke RR, Levy HL. Maternal phenylketonuria and hyperphenylalaninemia. An international survey of the outcome of untreated and treated pregnancies.[J]. N Engl J Med. 1980;303(21):1202-8.

6. Prick BW, Hop WC, Duvekot JJ. Maternal phenylketonuria and hyperphenylalaninemia in pregnancy: pregnancy complications and neonatal sequelae in untreated and treated pregnancies.[J]. Am J Clin Nutr. 2012;95(2):374-82.

7. Dent CE Etiologic Factors in Mental Retardation: Report of the 23rd Ross Pediatric Research Conference, 1956. Ross Laboratories; Columbus, $\mathrm{OH}: 1957$. The relation of biochemical abnormality to the development of mental defect in phenylketonuria; pp. 28-33.

8. Ross LF, Paul DB. 50 Years Ago in The Journal of Pediatrics: Maternal Phenylketonuria: Implications for Growth and Development. J Pediatr. 2018;201:121.

9. Listed N. Committee opinion no: 636: Management of women with phenylketonuria[J]. Obstetrics Gynecology. 2015;125(6):154850.

10. Widaman KF, Azen C. Relation of prenatal phenylalanine exposure to infant and childhood cognitive outcomes: results from the International Maternal PKU Collaborative Study[J]. Pediatrics. 2003;112(6 Pt 2):1537-43.

11. Cao Y. Yuan P, Wang YP. Mao M, Zhu J. The profile of newborn screening coverage in China. J Med Screen. 2009;16(4):163-6.

12. Zhong K. Wang W, He F. Wang Z. The status of neonatal screening in China, 2013. J Med Screen. 2016;23(2):59-61.

\section{Tables}

Table 1. Physiological reference values of Phe in AF

\begin{tabular}{|c|c|c|c|c|c|c|c|c|c|c|}
\hline Index & $\begin{array}{l}\text { Average } \\
(\mu \mathrm{mol} / \mathrm{L})\end{array}$ & $\begin{array}{l}\text { Minimum } \\
(\mu \mathrm{mol} / \mathrm{L})\end{array}$ & $\begin{array}{l}0.2 \% \\
(\mu \mathrm{mol} / \mathrm{L})\end{array}$ & $\begin{array}{l}0.5 \% \\
(\mu \mathrm{mol} / \mathrm{L})\end{array}$ & $\begin{array}{l}1.0 \% \\
(\mu \mathrm{mol} / \mathrm{L})\end{array}$ & $\begin{array}{l}50 \% \\
(\mu \mathrm{mol} / \mathrm{L})\end{array}$ & $\begin{array}{l}99 \% \\
(\mu \mathrm{mol} / \mathrm{L})\end{array}$ & $\begin{array}{l}99.5 \% \\
(\mu \mathrm{mol} / \mathrm{L})\end{array}$ & $\begin{array}{l}99.8 \% \\
(\mu \mathrm{mol} / \mathrm{L})\end{array}$ & $\begin{array}{l}\text { Maximum } \\
(\mu \mathrm{mol} / \mathrm{L})\end{array}$ \\
\hline Phe & 24.22 & 10.0 & 9.73 & 10.79 & 11.55 & 23.6 & 44.27 & 48.47 & 54.48 & 54.0 \\
\hline
\end{tabular}

\section{Figures}




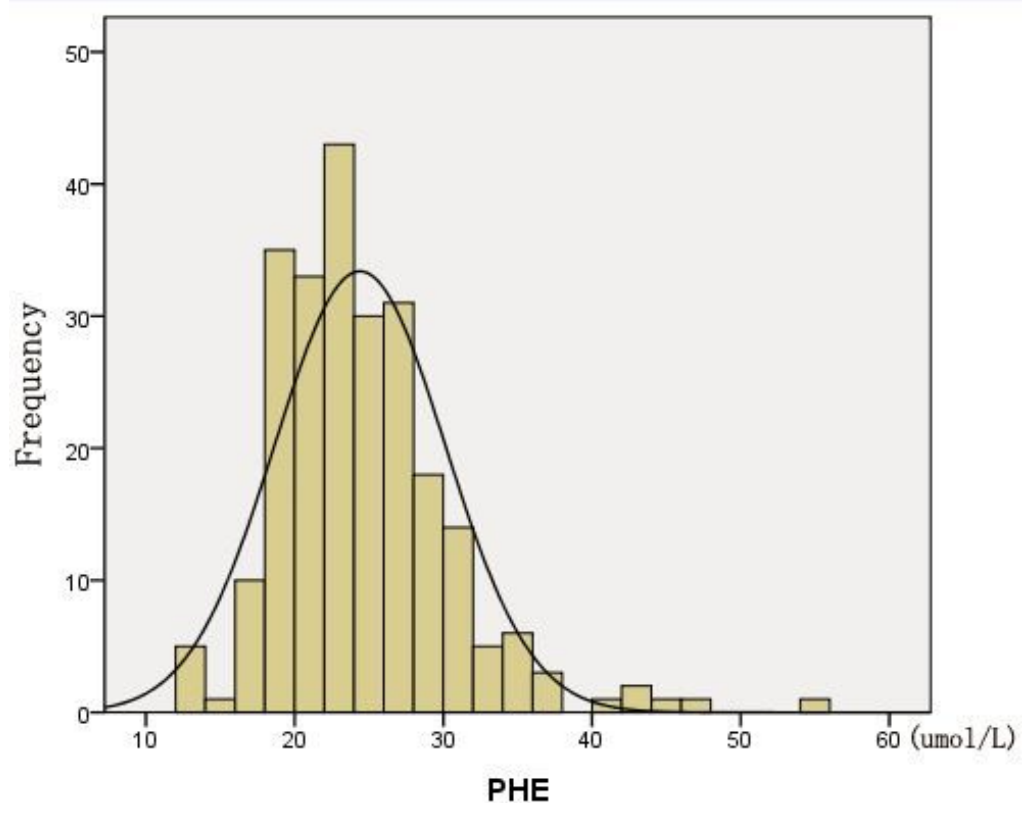

Figure 1

Frequency distribution histogram of Phe levels in AF of 345 pregnant women in the control group as detected by MS/MS.

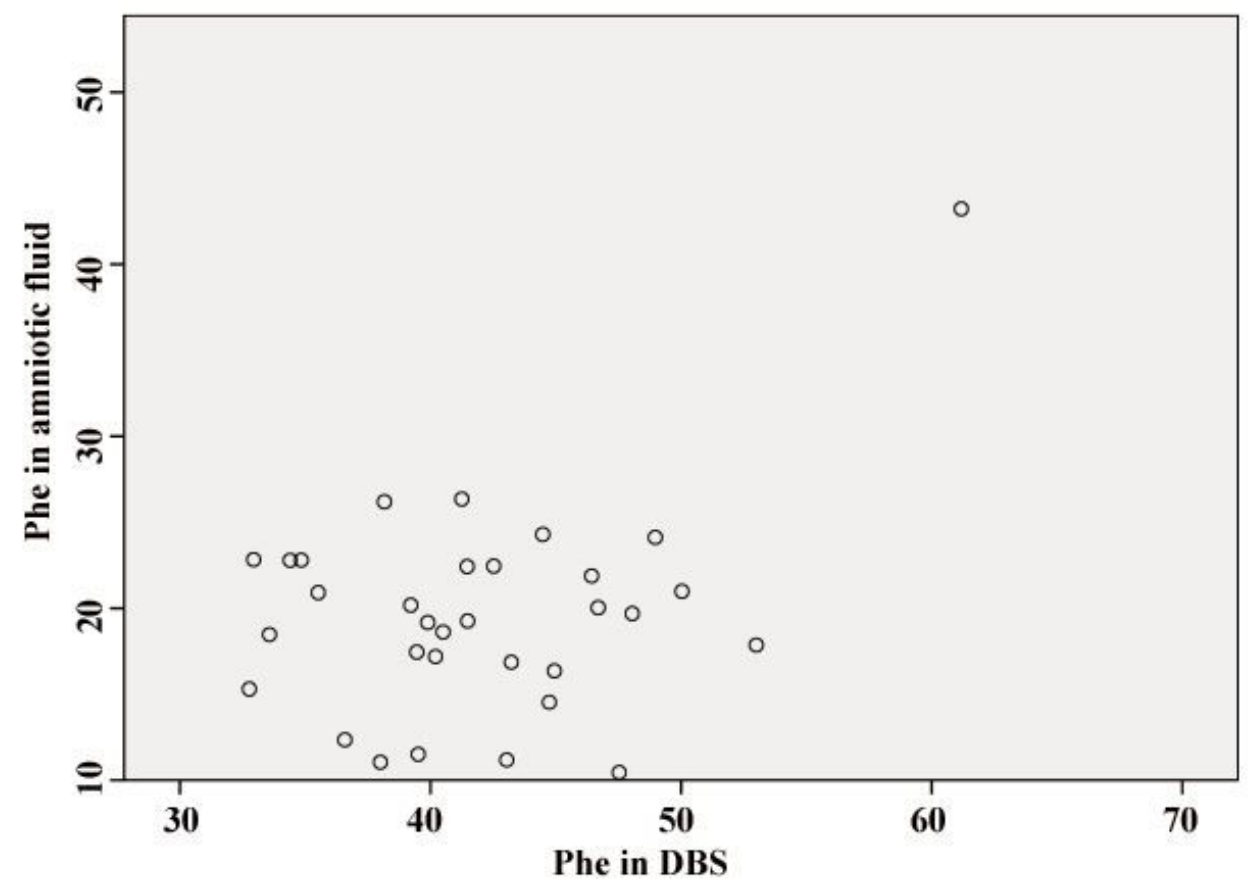

Figure 2

Correlation between Phe concentrations in AF and in DBSs as detected. by MS/MS. 